\title{
The trachea: The first tissue-engineered organ?
}

\author{
Pierre R. Delaere, MD, PhD, ${ }^{a}$ and Dirk Van Raemdonck, $\mathrm{MD}, \mathrm{PhD}^{\mathrm{b}}$
}

Major medical breakthroughs deserve the necessary press attention to inform the medical community and public of the news. Unfortunately, misrepresentation of medical information can occur and is particularly problematic when members of the professional and public press are misled to believe unrealistic medical breakthroughs.

In 2008 and 2011, 2 revolutionary articles were published in The Lancet reporting on regeneration of a tracheal transplant. ${ }^{1,2}$ The tracheal transplant procedure used stem cells extracted from the patient's bone marrow in conjunction with a synthetic scaffold to create the artificial organ. In September 2012, an article printed in The New York Times, "A First: Organs Tailor-Made With Body's Own Cells," ${ }^{3}$ recognized tracheal regeneration as the first regenerative medicine procedure designed to implant "bioartificial" organs. This achievement was touted as the beginning of complex organ engineering for the heart, liver, and kidneys, and it was suggested that allotransplantation along with immunosuppression might become problems of the past.

Two questions, however, remain: (1) How does a synthetic tube transform into a viable airway tube? (2) Is the trachea really the first bioengineered organ?

\section{REGENERATION VERSUS SECONDARY WOUND HEALING}

In reports on bioengineered tracheas, it is frequently stated that the trachea is well suited to tissue engineering because it consists of a hollow, relatively simple structure. In reality, however, the trachea has an ingenious morphology that allows it to fulfill its function (Figure 1, $A$ ). It is thus one of the most difficult organs of the human body to replace. ${ }^{4}$ To date, regeneration within the airway tract is only possible for epithelial defects above the basement membrane. If a tissue injury is severe and involves damage to both epithelial cells and the submucosal layer, healing will be accomplished by secondary intention, leading to stenosis of the airway (Figure 1, B). The current challenge for regenerative medicine is to overcome

\footnotetext{
From the Departments of Otolaryngology Head \& Neck Surgery a and Thoracic Surgery, ${ }^{\mathrm{b}}$ University Hospital Leuven, Leuven, Belgium.

Disclosures: Authors have nothing to disclose with regard to commercial support.

Received for publication Nov 3, 2013; revisions received Nov 24, 2013; accepted for publication Dec 20, 2013; available ahead of print Feb 4, 2014.

Address for reprints: Pierre R. Delaere, MD, PhD, Department of ENT, Head \& Neck Surgery, University Hospital Leuven, Kapucijnenvoer 33, 3000 Leuven, Belgium (E-mail: Pierre.Delaere@uzleuven.be).

J Thorac Cardiovasc Surg 2014;147:1128-32

0022-5223/\$36.00

Copyright (c) 2014 by The American Association for Thoracic Surgery

http://dx.doi.org/10.1016/j.jtcvs.2013.12.024
}

barriers to regeneration of the epithelial lining in full-thickness mucosal defects, which is not yet possible (Figure 1, C).

\section{PROSTHETIC VERSUS REGENERATED TRACHEA}

Tracheal regeneration for airway repair with bioengineered constructs typically consists of a cartilaginous tube lined with viable respiratory mucosa. ${ }^{1,2}$ These constructs are created with bone marrow stem cells and growth factors (of which the clinical value for tissue healing is unknown) that are applied to a nonvascularized scaffold. The mechanism behind the transformation from nonviable construct to viable airway cannot be explained with our current knowledge of tissue healing, tissue transplantation, and tissue regeneration. In fact, cells have never been observed to adhere, grow, and regenerate into complex tissues when applied to an avascular or synthetic scaffold. Moreover, this advanced form of tissue regeneration has never been observed in laboratory-based research.

Of the 14 patients who have received bioengineered tracheas,,$^{5} 3$ have been described in case reports in 4 different articles (the first patient was described after both 6-month and 5-year follow-ups). ${ }^{1,2,6,7}$ In these articles, 2 different scaffolds ( 2 cases with an enzymatically decellularized tracheal allotransplant ${ }^{1,6,7}$ and 1 case with a synthetic nanocomposite ${ }^{2}$ ) were reported as being used, along with 2 different techniques of applying bone marrow cells to the scaffold ( 2 cases with a bioreactor for "ex vivo" use $^{1,2,7}$ and 1 case where the bone marrow cells were applied "in vivo" ${ }^{\circ}$ ). Production of the bioengineered trachea in all cases produced similar results, and the different approaches worked in comparable ways. Information gathered from published reports on these 3 patients and unpublished reports on an additional 11 patients suggests that mortality and morbidity were very high. ${ }^{5}$ More than half of the patients died within a 3-month period, and the patients who survived longer functioned with an airway stent that preserved the airway lumen.

From this information, it can be concluded that the bioengineered tracheal replacements were in fact airway replacements that functioned only as scaffolds, behaving in a similar way to synthetic tracheal prostheses. In recent years, most synthetic materials used for tracheal replacement have been tested in experimental animal research. From these studies, it became clear that definitive prosthetic replacement of the airway wall is not possible. ${ }^{8}$ To date, nearly all surgical prostheses that have been successful were observed in potentially sterile 


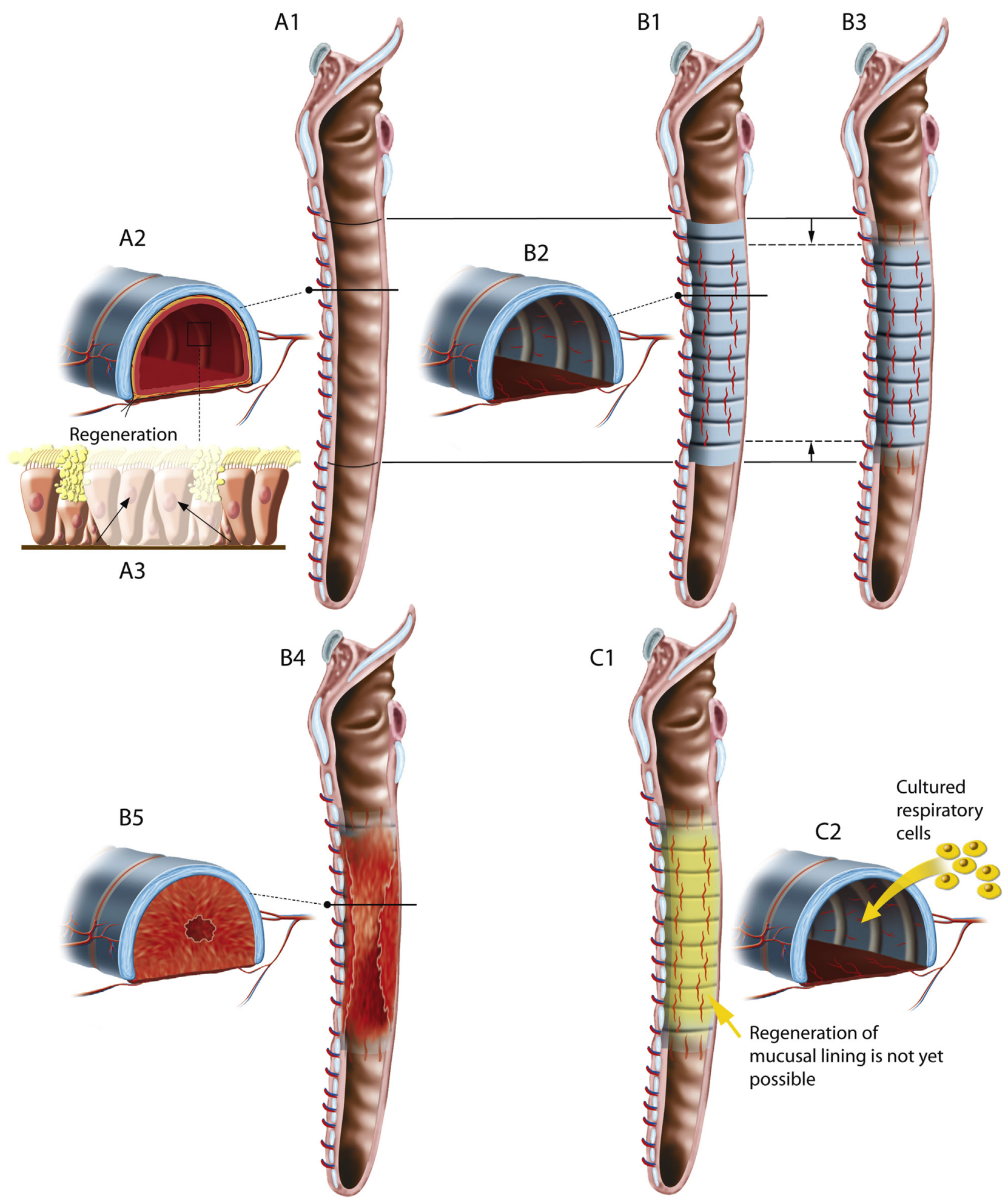

FIGURE 1. Wound healing of a tracheal mucosal defect. A, The trachea has a segmental blood supply that reaches the mucosal lining through the intercartilaginous ligaments and the membranous trachea (A1 and A2). The basement membrane of the mucosal layer supports a pseudostratified epithelium, the surface layer of which is columnar and ciliated, with deeper layers of basal cells. A superficial epithelial wound can heal through regeneration of the surface epithelium (A3). B, The healing pattern of a circumferential full-thickness mucosal defect (B1 and B2) is shown. Healing of full-thickness mucosal wounds combines granulation tissue formation, wound contraction, and reepithelialization from the wound edges (arrows). Airway stenosis in the middle part of the denuded segment can develop from circumferential wounds. Healing of anastomotic sites can enable several millimeters of respiratory epithelium to grow into the wound margins (B3) while the remaining tissue between those margins is left as uncovered granular tissue (B4 and B5). C, Research in tracheal tissue engineering should concentrate on attempts to regenerate the mucosal lining by applying epithelial stem cells $(\mathrm{C} 1$ and $\mathrm{C} 2)$ to a tracheal segment with a preserved blood supply and cartilaginous support. 

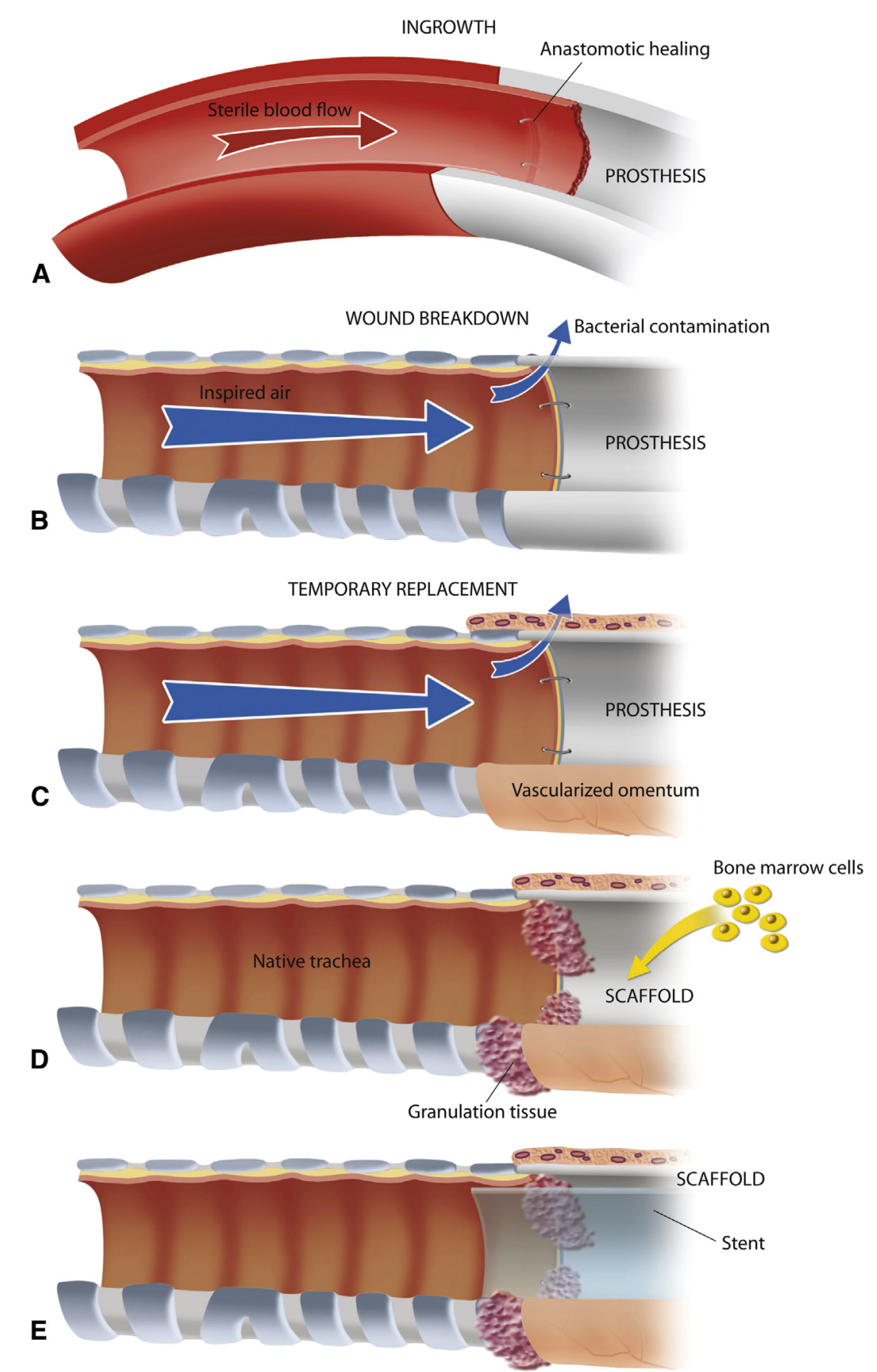

FIGURE 2. Prosthetic versus regenerated trachea A, Blood vessel prosthesis. Endothelialization of the luminal surface of vascular grafts occurs only 1 to 2 $\mathrm{cm}$ into the graft from the anastomotic site. These endothelial cells are derived from adjacent, native arterial endothelium, and they enable the anastomosis to heal. B, In the respiratory tract, the flow of inspired air will lead to bacterial contamination and wound breakdown at the anastomoses. The respiratory epithelium will not grow over the prosthesis-airway anastomosis. C, Airway prosthesis wrapped in vascularized tissue. A prosthesis may act as a temporary airway stent when it is wrapped by well-vascularized tissue (eg, omentum). The vascularized tissue around the prosthesis can temporary avoid the complications of wound breakdown at the anastomotic sites. D, A scaffold soaked in bone marrow cells will behave in similarly to a prosthesis. Granulation tissue at the anastomoses will be formed in patients surviving for longer periods. E, A stent can be placed as a temporary airway.

mesenchymal tissues. No example of successful prosthetic repair can be cited in the respiratory, gastrointestinal, or genitourinary tract. The internal site of the airway tract belongs to the outside world, and bacterial contamination at the interface between the airway and prosthesis prevent its ingrowth (Figure 2). The complications of 


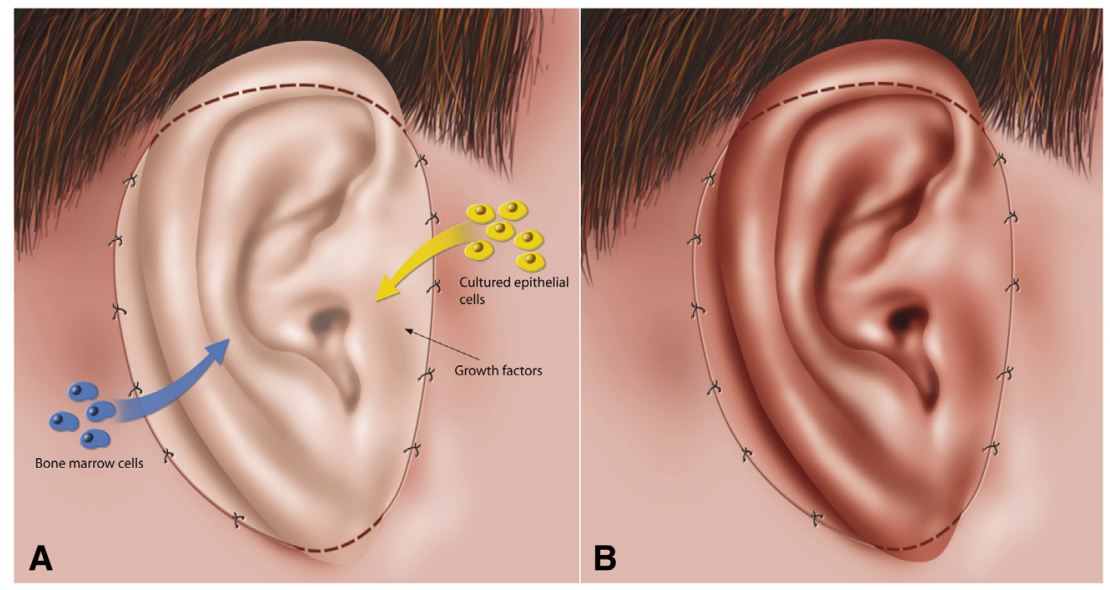

FIGURE 3. Visualization of the bioengineered tissue approach when applied to an external ear (theoretic example). A, The external ear has a comparable tissue composition (cartilage framework and epithelial lining) to the trachea. Here, an enzymatically decellularized allograft treated with bone marrow cells and cultured epithelial cells is attached to a recipient area without restoration of the blood supply. B, Direct visualization of the healing and revascularization of the bioengineered ear should be mandatory to prove (the hypothetical) success of the bioengineering process.

wound breakdown at the anastomoses can be temporarily delayed by wrapping the prosthesis in vascularized tissue, mostly transposed omentum. This is exactly what occurred in nearly all cases of the supposedly bioengineered tracheal replacements. The scaffolds were wrapped in vascularized omentum so that inevitable complications could be delayed. In patients who survive for longer periods, granulation tissue will form at the suture lines, a complication that necessitates placement of a stent to preserve the airway lumen temporarily (Figure 2,E).

\section{VISUALIZATION OF BIOENGINEERED TISSUE}

It seems contradictory that one of the most difficult organs to replace has been reported as being the first bioengineered organ. How can a synthetic tracheal replacement, destined to fail, be described as a major breakthrough in organ tissue engineering?

The dissemination of misleading information regarding bioengineering of complex tissues would not be possible in tissues that can be directly visualized (Figure 3). For a tracheal replacement, direct visualization of the transplant and subsequent healing is only possible in an experimental setting and during autopsy. Neither of these methods, however, were used to document the tissue-engineered tracheas in the 14 previous patients. Tracheal bioengineering was not tested in animal models, and despite the fact that several patients died after having received the bioengineered tracheal replacement, no postmortem histologic data have been published. Although in vivo visualization of tracheal transplants and their healing process is possible with serial computed tomographic scanning and endoscopic imaging performed at regular intervals after implantation, clear visualization of the transplant is lacking in all reports regarding the bioengineered trachea. Published computed tomographic images and endoscopic views show only images produced shortly after transplantation and either do not allow the clear visualization of the transplant or were obtained with an airway stent in place. ${ }^{1,2,6,7}$ In an editorial in Science discussing the controversy of this topic, ${ }^{5}$ the main scientist behind the bioengineering approach acknowledged that "it was difficult to tell what exactly had happened inside the implanted tracheas," which is remarkable when considering the high-quality computed tomographic scans and endoscopic approaches that are currently available.

The media hype regarding "stem cells" and the work done on an airway that is less accessible for direct visualization are key elements that explain publication of these papers in highly ranked medical journals. ${ }^{1,2,6,7,9}$

In conclusion, the ethical justification of tracheal replacement with a synthetic prosthesis or with a decellularized allograft in humans is questionable, because there are no available experimental data describing a possible successful outcome. The currently available published articles on bioengineered tracheas and the resulting media attention endanger the field of tracheal replacement and the field of tissue engineering as a whole. For patient safety, tracheal bioengineering must be demonstrated as being efficacious and safe before further transplants.

\section{References}

1. Macchiarini P, Jungebluth P, Go T, Asnaghi MA, Rees LE, Cogan T, et al Clinical transplantation of a tissue-engineered airway. Lancet. 2008;372: 2023-30. Erratum in: Lancet, 2009 Lancet. 2009;373:462.

2. Jungebluth P, Alici E, Baiguera S, Le Blanc K, Blomberg P, Bozóky B, et al Tracheobronchial transplantation with a stem-cell-seeded bioartificial nanocomposite: a proof-of-concept study. Lancet. 2011;379:1997-2004.

3. Fountain H. A first: organs tailor-made with body's own cells. The New York Times. September 15, 2012:A1. Available at: http://www.nytimes.com/2012/ 09/16/health/research/scientists-make-progress-in-tailor-made-organs.html?_r=0. Accessed September 15, 2012. 
4. Delaere P, Vranckx J, Verleden G, De Leyn P, Van Raemdonck D. Leuven Tracheal Transplant Group. Tracheal allotransplantation after withdrawal of immunosuppressive therapy. N Engl J Med. 2010;362:138-45.

5. Vogel G. Trachea transplants test the limits. Science. 2013;340:266-8.

6. Elliott MJ, De Coppi P, Speggiorin S, Roebuck D, Butler CR, Samuel E, et al. Stem-cell-based, tissue engineered tracheal replacement in a child: a 2-year follow-up study. Lancet. 2012;380:994-1000.
7. Gonfiotti A, Jaus MO, Barale S, Baiguera S, Comin C, Lavorini F, et al The first tissue-engineered airway transplantation: 5-year follow-up results Lancet. 2014;383:238-44.

8. Grillo HC. Tracheal replacement: a critical review. Ann Thorac Surg. 2002;73: 1995-2004.

9. Jungebluth P, Moll G, Baiguera S, Macchiarini P. Tissue-engineered airway: a regenerative solution. Clin Pharmacol Ther. 2012;91:81-93. 